\title{
Exosomes derived from oxidized LDL-stimulated macrophages attenuate the growth and tube formation of endothelial cells
}

\author{
CHAOYANG HUANG, YUAN HUANG, YIJIANG ZHOU, WENCHENG NIE, \\ XIANGYUAN PU, XIAOLEI XU and JIANHUA ZHU \\ Department of Cardiology, The First Affiliated Hospital, \\ Zhejiang University School of Medicine, Hangzhou, Zhejiang 310003, P.R. China
}

Received April 14, 2017; Accepted November 2, 2017

DOI: $10.3892 / \mathrm{mmr} .2018 .8380$

\begin{abstract}
Oxidized low-density lipoprotein (oxLDL) has a critical role in the development of atherosclerosis. The participation of oxLDL-stimulated macrophages has been well-established in atherosclerosis, however the underlying mechanisms are unclear. Macrophage-derived exosomes are actively released and are involved in numerous physiological and pathological processes. However, the function of exosomes secreted by oxLDL-stimulated macrophages in atherosclerosis remains unknown. Exosomes from oxLDL-treated macrophages and controls were co-cultured with endothelial cells and the exosomes were taken up by endocytosis. Cell Counting Kit- 8 and tube formation assay results revealed that exosomes derived from oxLDL-stimulated macrophages reduced the growth and tube formation ability of endothelial cells. Suppression of exosomal secretion by oxLDL-stimulated macrophages rescued the growth and tube formation ability of endothelial cells. Therefore, the results of the present study indicate that oxLDL-stimulated macrophages may attenuate the growth and tube formation of endothelial cells, at least in part through exosomal transfer. This may provide novel targets for the development of atherosclerosis therapeutics.
\end{abstract}

\section{Introduction}

Atherosclerosis is a disease in which the inside of an artery narrows due to the build-up of plaque. Atherosclerosis may result in the development of cardiovascular disease and is a leading cause of morbidity and mortality in developed countries $(1,2)$. Atherosclerotic lesions are characterized by a thin fibrous cap, large lipid core and the accumulation of

Correspondence to: Dr Chaoyang Huang, Department of Cardiology, The First Affiliated Hospital, Zhejiang University School of Medicine, 79 Qingchun Road, Hangzhou, Zhejiang 310003, P.R. China

E-mail: huangchaoyang75@126.com

Key words: atherosclerosis, oxidized low-density lipoprotein, exosome, macrophage, endothelial cells macrophages. Lesions with abundant macrophage accumulation are referred to as 'vulnerable plaques' (3), as these lesions are susceptible to rupture, which results in thrombosis and eventually leads to myocardial and cerebral infarction (4). Endothelial function impairment is a primary pathological factor in the development of atherosclerotic lesions (5). Oxidative stress, endoplasmic reticulum (ER) stress and inflammation have emerged as primary contributors to endothelial cell dysfunction (6-8).

Oxidized low-density lipoprotein (oxLDL) results from one of the biologically relevant modifications in LDL in the form of oxidation, since LDL particles are markedly sensitive to oxidative damage. oxLDL accumulation is one of the numerous causes of atherosclerosis initiation and progression (9). Initially, oxLDL binds to scavenger receptors on the vascular endothelial cell surface, inducing adhesion molecule expression that facilitates monocyte adhesion and migration into the sub-endothelial layer (10). OxLDL transverses the endothelial barrier and enters the sub-endothelium, where it induces the differentiation of monocytes into macrophages, which attempt to phagocytose the oxLDL and become lipid-laden foam cells (11). The latter contributes to the expansion of the atherosclerotic plaque lipid core and exacerbates oxidative stress (12). Macrophage accumulation is regarded as a crucial step in atherosclerotic plaque formation and development. However, the interactions that occur between macrophages and endothelial cells in atherosclerosis are still unclear.

Exosomes are nano-sized membrane vesicles with a diameter of 30-100 nm (13-15). Macrophage-derived exosomes may regulate the cellular functions of fibroblasts, vascular smooth muscle and endothelial cells through the delivery of microRNAs (miRNAs), mRNAs and proteins (16-19). However, the function of exosomes secreted by oxLDL-stimulated macrophages in atherosclerosis is yet to be elucidated.

The present study demonstrated that oxLDL-stimulated macrophages attenuated the growth and tube formation of endothelial cells, in part through exosomal transfer. Exosomes from oxLDL-treated macrophages and comparable controls were collected and co-cultured with endothelial cells and the exosomes were subsequently endocytosed. Cell Counting Kit-8 (CCK8) and tube formation assays revealed that exosomes derived from oxLDL-stimulated macrophages reduced the 
growth and tube formation abilities of endothelial cells, compared with control macrophages. Suppression of exosome secretion by oxLDL-stimulated macrophages rescued the growth and tube formation abilities of endothelial cells. The results indicated that oxLDL-stimulated macrophages attenuated the growth and tube formation of endothelial cells, partially through exosomal transfer. This may provide novel targets for atherosclerosis therapy.

\section{Materials and methods}

Cell culture. THP-1 human monocytic cells were purchased from American Type Culture Collection (ATCC; Manassas, VA, USA) and maintained in RPMI-1640 medium (Thermo Fisher Scientific Inc., Waltham, MA, USA) and human umbilical vein endothelial cells (HUVECs) were purchased from ATCC and maintained in Dulbecco's modified Eagle's medium(DMEM)/F12 (Thermo Fisher Scientific Inc.). The two media were supplemented with $10 \%$ heat-inactivated fetal bovine serum (Invitrogen; Thermo Fisher Scientific Inc.), 100 units $/ \mathrm{ml}$ penicillin and $100 \mu \mathrm{g} / \mathrm{ml}$ streptomycin in a humidified atmosphere of $5 \% \mathrm{CO}_{2}$ at $37^{\circ} \mathrm{C}$. All cells were confirmed to be free of mycoplasma contamination.

Electron microscopic observation of exosomes. The exosome suspension was added to an equal volume of $4 \%$ paraformaldehyde (Nacalai Tesque, Inc., Kyoto, Japan), and the mixture was applied to a Formvar/Carbon film-coated transmission electron microscope (TEM) grid (Alliance Biosystems, Osaka, Japan). Then, the sample was fixed by incubation with $1 \%$ glutaraldehyde for $5 \mathrm{~min}$, washed with PBS, and incubated with $1 \%$ uranyl acetate for $5 \mathrm{~min}$ at $4^{\circ} \mathrm{C}$. The sample was observed under a TEM (Hitachi H7650; Hitachi, Ltd., Tokyo, Japan).

Exosome isolation and co-culture with HUVECs. To isolate the exosomes, THP-1 exosomes were first treated with $50 \mu \mathrm{g} / \mathrm{ml}$ oxLDL (Shanghai Leuven Biotechnology Co., Ltd., Shanghai, China) for $48 \mathrm{~h}$. The supernatant was subsequently collected and centrifuged at $1,000 \mathrm{x}$ g for $10 \mathrm{~min}$, then $3,000 \mathrm{x} \mathrm{g}$ for $30 \mathrm{~min}$ at $4^{\circ} \mathrm{C}$ to remove cell components and fragments. A total exosome isolation kit (Thermo Fisher Scientific Inc.) was then added to the sample overnight at $4^{\circ} \mathrm{C}$, prior to centrifugation at $10,000 \mathrm{xg}$ for $1 \mathrm{~h}$ at $4^{\circ} \mathrm{C}$. Exosomes were re-suspended in PBS and stored at $-80^{\circ} \mathrm{C}$. The exosome concentration was detected using a bicinchoninic acid protein assay kit (Beyotime Institute of Biotechnology, Hangzhou, China). Exosomes were then co-cultured with $10^{5}$ HUVECs in $50 \mathrm{ng} / \mathrm{ml}$ DMEM for $24 \mathrm{~h}$ at $37^{\circ} \mathrm{C}$.

Western blot analysis. Western blot analysis was performed to analyse the expression of the exosomal marker cluster of differentiation (CD)63. Cells were lysed with radioimmunoprecipitation assay buffer (50 mM Tris-HCl, pH 7.5; $150 \mathrm{mM} \mathrm{NaCl}$; $1 \%$ Triton X-100 and 0.5\% sodium deoxycholate) and blocked with the complete mini protease inhibitor cocktail $30 \mathrm{~min}$ at $4^{\circ} \mathrm{C}$ (Roche Diagnostics GmbH, Mannheim, Germany). The protein concentration was detected using a bicinchoninic acid protein kit (Beyotime Institute of Biotechnology). A total of 20-30 $\mu \mathrm{g}$ lysate samples were subsequently separated on
8-12\% SDS-PAGE gel and transferred onto polyvinylidene membranes. The membranes were incubated with the following primary antibodies overnight at $4^{\circ} \mathrm{C}$ : rabbit anti-human CD63 (cat. no. ab59479, 1:1,000; Abcam, Cambridge, UK), rabbit anti-human CD9 (cat. no. ab92726, 1:1,000; Abcam), mouse anti-human CD81 (cat. no. H00000975-B01P, 1:500; Novus Biologicals, LLC, Littleton, CO, USA) and mouse anti-Actin (cat. no. CP01-1EA, 1:10,000; Merck KGaA, Darmstadt, Germany). Membranes were then incubated with the following horse radish peroxidase (HRP)-conjugated anti-rabbit secondary antibody (cat. no. 7074; 1:10,000; CST Biological Reagents Co., Ltd., Shanghai, China) and HRP-anti-mouse antibody (cat. no. 7076; 1:10,000; CST Biological Reagents Co., Ltd.) at room temperature for $1 \mathrm{~h}$. The antibodies were detected using an enhanced chemiluminescence kit (cat. no. PI32209; Pierce; Thermo Fisher Scientific Inc.).

PHK67 stained exosomes co-culture with HUVECs. Purified exosomes stained with PHK67 (Sigma-Aldrich; Merck KGaA, Darmstadt, Germany) according to the manufacture's protocols. Briefly, exosomes resuspended in a buffer provided in the kits were mixed with the PKH dyes and were incubated for $5 \mathrm{~min}$ at room temperature. Next, the samples were added to PBS supplemented with $5 \%$ bovine serum albumin and were ultracentrifuged at $100,000 \mathrm{~g}$ for $1 \mathrm{~h}$ at $4{ }^{\circ} \mathrm{C}$ to remove free dyes. Then, the stained exosomes were co-cultured with HUVECs which were then fixed with $4 \%$ paraformaldehyde at $25^{\circ} \mathrm{C}$ for $20 \mathrm{~min}$.

GW4869 treatment of macrophages. Macrophages were

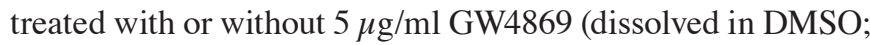
MedChem Express, USA) for $24 \mathrm{~h}$ at $37^{\circ} \mathrm{C}$ to inhibit exosome secretion.

CCK8 assay. The CCK8 assay was conducted according to the manufacturer's protocol (CK04; Dojindo Molecular Technologies, Inc., Kumamoto, Japan). Exosome-treated cells were plated at equal cell density $(2,000$ cells/100 $\mu \mathrm{l}$ well $)$ in 96-well plates with $300 \mu \mathrm{g} / \mathrm{ml}$ cetuximab (Merck KGaA) for continuous detection over a 5-day period. At the beginning of the second day, the culture was terminated with the addition of $10 \mu \mathrm{l} \mathrm{CCK} 8(5 \mathrm{mg} / \mathrm{ml})$ to the original culture medium. Following $2 \mathrm{~h}$, plates were measured using a microplate reader (Biotek Elx800, Biotek Instruments, Inc., Winooski, VT, USA). Cell proliferation was measured using optical density at $450 \mathrm{~nm}$.

Tube formation assay. The exosome-treated HUVECs ( $5 \times 10^{4} / 96$ well plate) were re-suspended in the conditioned medium and seeded into growth factor-reduced Matrigel (356234; BD Biosciences, Franklin Lakes, NJ, USA). Following incubation for $6 \mathrm{~h}$ images of the tubes formed were captured under an inverted microscope at x100 magnification. The length of tubes was measured using Image J software version 1.47 (National Institutes of Health, Bethesda, MD, USA).

Statistical analysis. Statistical analysis was performed using the Student's t-test to compare between groups. Data were presented as mean \pm standard error of the mean. A Tukey test was conducted for multiple comparisons in conjunction with one way analysis of variance. $\mathrm{P}<0.05$ was considered to 
A

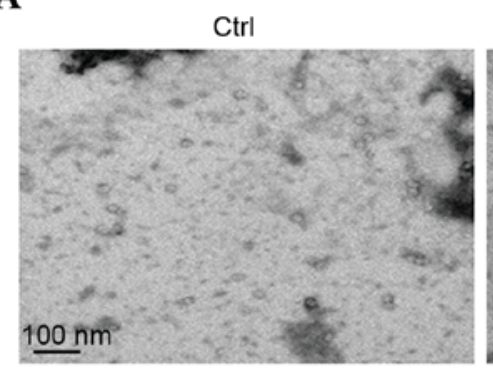

oxLDL-stimulated

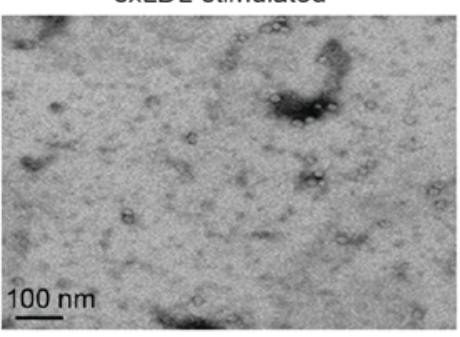

B

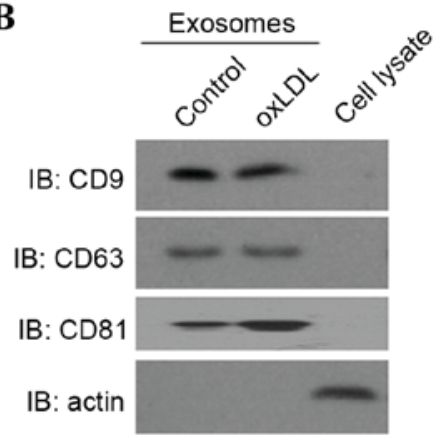

Figure 1. Exosomes derived from oxLDL-stimulated macrophages. (A) Representative transmission electron micrograph revealing the typical morphology and size of exosomes isolated from oxLDL-stimulated or non-stimulated macrophages (50-150 nm). Scale bar, $100 \mathrm{~nm}$. (B) Western blot analysis identified the presence of the exosomal markers CD9, CD63 and CD81 and the absence of actin in exosomes derived from oxLDL-stimulated or non-stimulated macrophages. oxLDL, oxidised low-density lipoprotein; IB, immunoblotting; CD, cluster of differentiation; Ctrl, control.

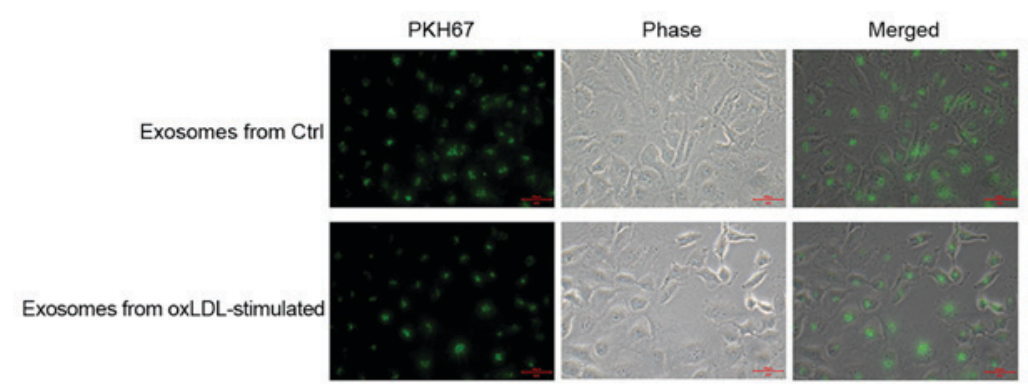

Figure 2. Endothelial cells endocytose exosomes derived from oxLDL-stimulated macrophages. Fluorescent microscopy results of exosomes isolated from oxLDL-stimulated or non-stimulated macrophages co-cultured with endothelial cells. Exosomes were stained with PHK67 (green). Magnification, $400 x$. oxLDL, oxidised low-density lipoprotein; Ctrl, control.

indicate a statistically significant difference. SPSS version 13.0 was used for analysis (SPSS, Inc., Chicago, IL, USA).

\section{Results}

Exosomes derived from oxLDL-stimulated macrophages. To investigate the function of oxLDL-stimulated macrophage-derived exosomes in atherosclerosis, exosomes were isolated from oxLDL-treated or untreated THP-1 cells. The morphology of exosomes collected was observed with scanning electron microscopy. The diameters of the exosomes ranged from 30-120 nm (Fig. 1A). Western blot analysis revealed that exosomes were enriched with the exosomal markers CD9, CD63 and CD81 whereas actin was enriched in the cellular lysate (Fig. 1B), indicating an effective isolation of exosomes.

Endothelial cells endocytose exosomes derived from oxLDL-stimulated macrophages. PHK67 labeled exosomes were co-cultured with HUVECs. Following $24 \mathrm{~h}$, co-cultured HUVECs were fixed and observed by fluorescent microscopy. Exosomes were observed inside the co-cultured HUVECs (Fig. 2), verifying that endothelial cells endocytosed the exosomes derived from oxLDL-stimulated macrophages.

Exosomes derived from oxLDL-stimulated macrophages reduce the growth and tube formation abilities of endothelial cells. The growth and tube formation abilities of HUVECs co-cultured with exosomes isolated from oxLDL-treated or untreated macrophages were analysed to explore the effects of oxLDL-stimulated macrophage-derived exosomes on endothelial cells. CCK8 assay results revealed that oxLDL-stimulated macrophage-derived exosomes decreased the cell growth ability of HUVECs significantly, compared with exosomes isolated from untreated macrophages (Fig. 3A). Furthermore, tube formation assay results demonstrated that incubation with oxLDL-stimulated macrophage-derived exosomes reduced the tube formation ability of HUVECs compared with to the untreated control (Fig. 3B). HUVECs were also treated with $50 \mathrm{ng} / \mathrm{ml}$ oxLDL instead of exosomes. The CCK8 assay and tube formation assay results revealed that $50 \mathrm{ng} / \mathrm{ml}$ oxLDL treatment did not suppress the growth and tube formation (Fig. 3), indicating that specifically the exosomes derived from oxLDL-stimulated macrophages reduced the growth and tube formation abilities of endothelial cells.

Suppression of exosomal secretion in oxLDL-stimulated macrophages rescues the growth and tube formation abilities of endothelial cells. To further verify the effects of oxLDL-stimulated macrophage-derived exosomes on endothelial cells, exosome generation was blocked with exosomal release inhibitors GW4869 $(20,21)$ (Fig.4A). CCK8 assay results revealed that exosomes collected from oxLDL-stimulated macrophages treated with the GW4869 inhibitor recovered the cell growth ability of HUVECs compared with the dimethyl 
A

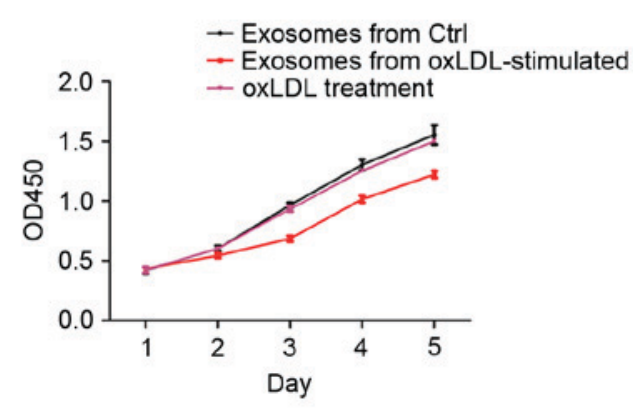

B

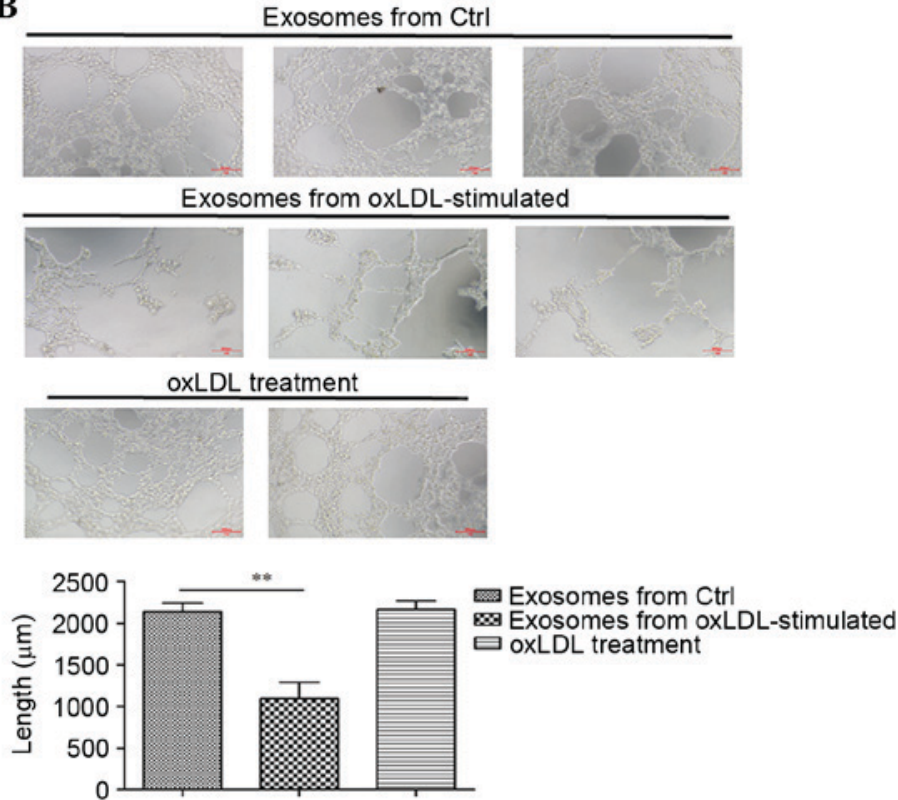

Figure 3. Exosomes derived from oxLDL-stimulated macrophages reduce the growth and tube formation abilities of endothelial cells. (A) Cell counting kit 8 analysis results revealed that HUVECs treated with exosomes isolated from oxLDL-stimulated macrophages had a reduced rate of proliferation in comparison with the untreated control $(\mathrm{P}<0.0001) ; 50 \mathrm{ng} / \mathrm{ml}$ oxLDL treatment alone did not affect the proliferation rate. (B) Tube formation analysis revealed that the tube formation ability of HUVECs treated with exosomes isolated from oxLDL-stimulated macrophages was reduced in comparison with the untreated control $\left({ }^{* *} \mathrm{P}<0.0001\right) .50 \mathrm{ng} / \mathrm{ml}$ oxLDL treatment did not affect tube formation. oxLDL, oxidised low-density lipoprotein; OD, optical density; HUVECs, human umbilical vein endothelial cells; Ctrl, control.

A

: CD9

IB: CD63

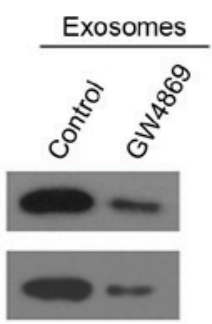

B

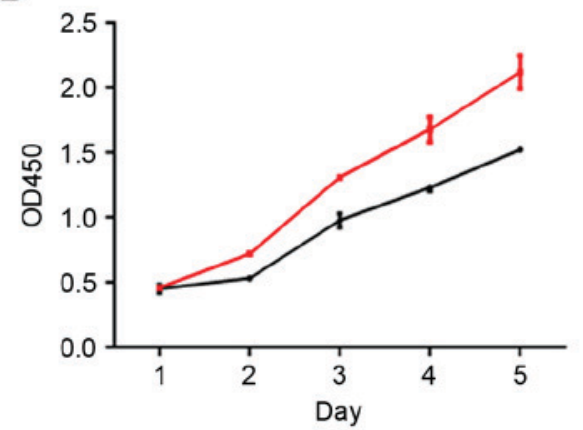

\section{$\rightarrow$ Exosomes from Ctrl \\ - Exosomes from GW4869 treated}

C

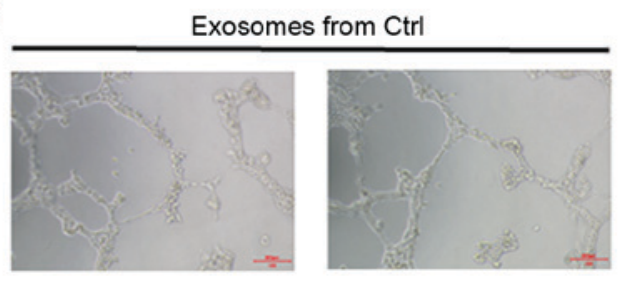

Exosomes from GW4869 treated
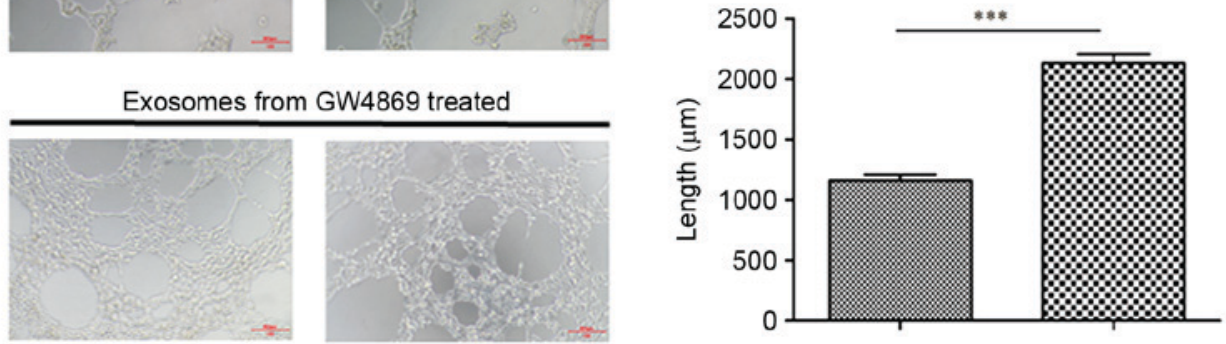

Figure 4. Inhibition of exosome secretion in oxLDL-stimulated macrophages rescues the growth and tube formation abilities of endothelial cells. (A) Western blot analysis of exosomal markers CD9 and CD63 verified that GW4869 treatment reduced exosome release. (B) Cell Counting Kit-8 analysis results revealed that GW4869 treatment rescued the proliferative ability of HUVECs co-cultured with exosomes collected from oxLDL-stimulated macrophages in comparison with the DMSO treatment with oxLDL-stimulation ( $\mathrm{P}=0.0032$ ). (C) Tube formation analysis revealed that GW4869 treatment rescued the tube formation ability of HUVECs co-cultured with exosomes collected from oxLDL-stimulated macrophages in comparison with the DMSO treatment with oxLDL-stimulation ( $\left.{ }^{* * *} \mathrm{P}<0.0001\right)$. oxLDL, oxidised low-density lipoprotein; OD, optical density; HUVECs, human umbilical vein endothelial cells; Ctrl, control; IB, immunoblotting; $\mathrm{CD}$, cluster of differentiation. 
sulfoxide (DMSO) control (Fig. 4B). The tube formation assay results also indicated that the exosomes collected from the oxLDL-stimulated macrophages treated with the GW4869 inhibitor regained tube formation ability compared with the DMSO control (Fig. 4C). These results indicated that the blockade of exosomal secretion in oxLDL-stimulated macrophages rescued normal endothelial function.

\section{Discussion}

It has previously been demonstrated that exosomes mediate cell-cell communication through the transfer of RNAs and proteins, which contributes to a variety of physiological and pathological processes, including cardiovascular disease (22-24). The function of exosomes secreted by oxLDL-stimulated macrophages in atherosclerosis was investigated in the present study. Exosomes derived from oxLDL-stimulated macrophages were demonstrated to reduce the growth and tube formation abilities of endothelial cells. To the best of the author's knowledge, this is the first study to demonstrate that oxLDL-stimulated macrophage-derived exosomes mediate endothelial cell function.

OxLDL contributes to atherosclerotic plaque formation and progression through several mechanisms, including the formation of macrophage foam cells and the induction of endothelial cell activation and dysfunction $(25,26)$. OxLDL stimulates the differential regulation of acid sphingomyelinase in macrophages. Pro-inflammatory responses to oxLDL-immune complexes are mediated by the prolonged activation of acid sphingomyelinase (26). Furthermore, oxLDL-induced injury in retinal pigment epithelium enhances the exosomal and apoptotic bleb release of the membrane complement regulatory factors CD46 and CD59, indicating that oxLDL stimulation may alter the components of exosomal release (27). Macrophage-derived exosomes have a variety of functions in cardiovascular disease, including the suppression of fibroblast proliferation, the induction of inflammation and the activation of nuclear factor- $\kappa \mathrm{B}$ in endothelial cells (16). OxLDL-stimulated macrophage-derived exosomes were demonstrated to mediate the growth and tube formation abilities of endothelial cells in the present study. Blockade of exosomal secretion in oxLDL-stimulated macrophages rescued the growth and tube formation abilities of endothelial cells. However, the observed effects may be partially attributed to oxLDL precipitation. The inhibitor GW4869 also has additional effects to the inhibition of vesicle release. Therefore, further research is required to verify the proposed function of exosomes.

Reciprocal interactions between endothelial cells and macrophages are thought to occur through secreted microvesicles including exosomes in angiogenic vascular niches (28). Exosomes may contain mRNAs, non-coding RNAs and proteins. miRNA-containing microvesicles may regulate inflammation in atherosclerotic disease (29). The horizontal transfer of macrophage-derived exosomal miRNA-155 has been demonstrated during cardiac injury (16). Therefore, the content of exosomes derived from oxLDL-stimulated macrophages requires further investigation.

In conclusion, the results of the present study indicated that oxLDL-stimulated macrophages attenuated the growth and tube formation of endothelial cells, in part through exosomal transfer. This may aid in the discovery of novel targets for atherosclerosis therapy.

\section{Acknowledgements}

The present study was supported by Zhejiang Provincial Science Foundation of China (grant no. LY17H020003), and Zhejiang Medical Science \& Technology Project (grant no. 2015KYA070).

\section{References}

1. Wong ND: Epidemiological studies of CHD and the evolution of preventive cardiology. Nat Rev Cardiol 11: 276-289, 2014.

2. Thomas MR and Lip GY: Novel risk markers and risk assessments for cardiovascular disease. Circ Res 120: 133-149, 2017.

3. Rohwedder I, Montanez E, Beckmann K, Bengtsson E, Dunér P, Nilsson J, Soehnlein O and Fässler R: Plasma fibronectin deficiency impedes atherosclerosis progression and fibrous cap formation. EMBO Mol Med 4: 564-576, 2012.

4. Badimon L and Vilahur G: Thrombosis formation on atherosclerotic lesions and plaque rupture. J Intern Med 276: 618-632, 2014.

5. Eren E, Yilmaz $\mathrm{N}$ and Aydin O: Functionally defective high-density lipoprotein and paraoxonase: A couple for endothelial dysfunction in atherosclerosis. Cholesterol 2013: 792090, 2013.

6. Husain K, Hernandez W, Ansari RA and Ferder L: Inflammation, oxidative stress and renin angiotensin system in atherosclerosis. World J Biol Chem 6: 209-217, 2015.

7. Xu Y, Zhu J, Hu X, Wang C, Lu D, Gong C, Yang J and Zong L: CLIC1 inhibition attenuates vascular inflammation, oxidative stress, and endothelial injury. PLoS One 11: e0166790, 2016.

8. Zhu L, Jia F, Wei J, Yu Y, Yu T, Wang Y, Sun J and Luo G: Salidroside protects against homocysteine-induced injury in human umbilical vein endothelial cells via the regulation of endoplasmic reticulum stress. Cardiovasc Ther 35: 33-39, 2017.

9. Pirillo A, Norata GD and Catapano AL: LOX-1, OxLDL, and atherosclerosis, Mediators Inflamm 2013: 152786, 2013.

10. Li Z, Cheng J and Wang L: Edaravone attenuates monocyte adhesion to endothelial cells induced by oxidized low-density lipoprotein. Biochem Biophys Res Commun 466: 723-727, 2015.

11. Yu XH, Fu YC, Zhang DW, Yin K and Tang CK: Foam cells in atherosclerosis. Clin Chim Acta 424: 245-252, 2013.

12. Wallner S, Grandl M, Liebisch G, Peer M, Orsó E, Sigrüner A, Sobota A and Schmitz G: oxLDL and eLDL induced membrane microdomains in human macrophages. PLoS One 11: e0166798, 2016.

13. Lötvall J, Hill AF, Hochberg F, Buzás EI, Di Vizio D, Gardiner C, Gho YS, Kurochkin IV, Mathivanan S, Quesenberry P, et al: Minimal experimental requirements for definition of extracellular vesicles and their functions: A position statement from the international society for extracellular vesicles. J Extracell Vesicles 3: 26913, 2014.

14. Huber HJ and Holvoet P: Exosomes: Emerging roles in communication between blood cells and vascular tissues during atherosclerosis. Curr Opin Lipidol 26: 412-419, 2015.

15. EV-TRACK Consortium, Van Deun J, Mestdagh P, Agostinis P, Akay Ö, Anand S, Anckaert J, Martinez ZA, Baetens T, Beghein E, et al: EV-TRACK: Transparent reporting and centralizing knowledge in extracellular vesicle research. Nat Methods 14: 228-232, 2017.

16. Wang C, Zhang C, Liu L, A X, Chen B, Li Y and Du J: Macrophage-derived mir-155-containing exosomes suppress fibroblast proliferation and promote fibroblast inflammation during cardiac injury. Mol Ther 25: 192-204, 2017.

17. Osada-Oka M, Shiota M, Izumi Y, Nishiyama M, Tanaka M, Yamaguchi T, Sakurai E, Miura K and Iwao H: Macrophage-derived exosomes induce inflammatory factors in endothelial cells under hypertensive conditions. Hypertens Res 40: 353-360, 2017.

18. Niu C, Wang X, Zhao M, Cai T, Liu P, Li J, Willard B, Zu L, Zhou E, Li Y, et al: Macrophage foam cell-derived extracellular vesicles promote vascular smooth muscle cell migration and adhesion. J Am Heart Assoc 5: e004099, 2016. 
19. Tang N, Sun B, Gupta A, Rempel H and Pulliam L: Monocyte exosomes induce adhesion molecules and cytokines via activation of NF- $\kappa$ B in endothelial cells. FASEB J 30: 3097-3106, 2016.

20. Zhao L, Luo H, Li X, Li T, He J, Qi Q, Liu Y and Yu Z: Exosomes derived from human pulmonary artery endothelial cells shift the balance between proliferation and apoptosis of smooth muscle cells. Cardiology 137: 43-53, 2017.

21. Gao W, Liu H, Yuan J, Wu C, Huang D, Ma Y, Zhu J, Ma L, Guo J, Shi H, et al: Exosomes derived from mature dendritic cells increase endothelial inflammation and atherosclerosis via membrane TNF- $\alpha$ mediated NF- $\kappa$ B pathway. J Cell Mol Med 20: 2318-2327, 2016.

22. Shen J, Huang CK, Yu H, Shen B, Zhang Y, Liang Y, Li Z, Feng X, Zhao J, Duan L and Cai X: The role of exosomes in hepatitis, liver cirrhosis and hepatocellular carcinoma. J Cell Mol Med 21: 986-992, 2017.

23. Zhang $\mathrm{Y}, \mathrm{Hu} \mathrm{YW}$, Zheng $\mathrm{L}$ and Wang Q: Characteristics and roles of exosomes in cardiovascular disease. DNA Cell Biol 36 202-211, 2017.

24. Jiang XC and Gao JQ: Exosomes as novel bio-carriers for gene and drug delivery. Int J Pharm 521: 167-175, 2017.
25. Zhang J, Wang D and He S: Roles of antibody against oxygenized low density lipoprotein in atherosclerosis: Recent advances. Int J Clin Exp Med 8: 11922-11929, 2015.

26. Truman JP, Al Gadban MM, Smith KJ, Jenkins RW, Mayroo N Virella G, Lopes-Virella MF, Bielawska A, Hannun YA and Hammad SM: Differential regulation of acid sphingomyelinase in macrophages stimulated with oxidized low-density lipoprotein (LDL) and oxidized LDL immune complexes: Role in phagocytosis and cytokine release. Immunology 136: 30-45, 2012 .

27. Ebrahimi KB, Fijalkowski N, Cano M and Handa JT: Oxidized low-density-lipoprotein-induced injury in retinal pigment epithelium alters expression of the membrane complement regulatory factors CD46 and CD59 through exosomal and apoptotic bleb release. Adv Exp Med Biol 801: 259-265, 2014.

28. Baer C, Squadrito ML, Iruela-Arispe ML and De Palma M: Reciprocal interactions between endothelial cells and macrophages in angiogenic vascular niches. Exp Cell Res 319: 1626-1634, 2013

29. Hulsmans M and Holvoet P: MicroRNA-containing microvesicles regulating inflammation in association with atherosclerotic disease. Cardiovasc Res 100: 7-18, 2013. 